\title{
Incorporating prior knowledge to facilitate discoveries in a genome-wide association study on age-related macular degeneration
}

\author{
Wan-Yu Lin ${ }^{1}$, Wen-Chung Lee ${ }^{1,2^{*}}$
}

\begin{abstract}
Background: Substantial genotyping data produced by current high-throughput technologies have brought opportunities and difficulties. With the number of single-nucleotide polymorphisms (SNPs) going into millions comes the harsh challenge of multiple-testing adjustment. However, even with the false discovery rate (FDR) control approach, a genome-wide association study (GWAS) may still fall short of discovering any true positive gene, particularly when it has a relatively small sample size.
\end{abstract}

Findings: To counteract such a harsh multiple-testing penalty, in this report, we incorporate findings from previous linkage and association studies to re-analyze a GWAS on age-related macular degeneration. While previous Bonferroni correction and the traditional FDR approach detected only one significant SNP (rs380390), here we have been able to detect seven significant SNPs with an easy-to-implement prioritized subset analysis (PSA) with the overall FDR controlled at 0.05. These include SNPs within three genes: CFH, CFHR4, and SGCD.

Conclusions: Based on the success of this example, we advocate using the simple method of PSA to facilitate discoveries in future GWASs.

\section{Background}

Substantial genotyping data produced by current highthroughput technologies have brought opportunities and difficulties. High-density genotyping platforms have been developed in a hope that underlying disease-associated genes can be identified through denser and denser collections of single-nucleotide polymorphism (SNP) data. However with the number of SNPs going into millions comes the harsh challenge of multiple-testing adjustment. To counteract multiple-testing penalty incurred by testing such a large number of SNPs, some genome-wide association studies (GWASs) responded by taking a large sample size-with the number of study subjects soaring into thousands, tens of thousands, or even more [1].

There are two approaches for multiple-testing adjustments. One is controlling the family-wise error rate (FWER), the other is controlling the false discovery rate (FDR) [2,3]. The FWER is defined as the probability of at least one type I error. Among methods for controlling

\footnotetext{
* Correspondence: wenchung@ntu.edu.tw

'Graduate Institute of Epidemiology, College of Public Health, National Taiwan University, Taipei, Taiwan
}

FWER, the Bonferroni correction is the best known approach, although it is very conservative. Holm's stepdown procedure [4] is less conservative than the classical Bonferroni correction. The FWER can also be controlled by the resampling-based $P$-value adjustment procedure. Compared with controlling the FWER, controlling the FDR is usually a more powerful approach. However, even with the FDR approach, a GWAS may still fall short of discovering any true positive gene, particularly when it has a relatively small sample size. When testing simultaneously for a huge number of SNPs, even true positive SNPs would have difficulty in standing out among all the noise, based on a straight (and brutal) comparison of their $p$ values. GWAS on age-related macular degeneration (AMD) is a good example, and we will show this in this paper.

The above simple FDR approach has been further extended to dependent tests and to tests with prior information [5]. The false discovery control with $P$-value weighting $[5,6]$ can improve power when the assignment of weights (based on previous linkage evidence [6]) is adequate, but there is some power loss when the 
weights are poorly assigned. Sun et al.'s [7] stratified false discovery control is another approach. They partitioned all SNPs into two subsets based on minor-allele frequencies (MAFs), and then the FDR control is applied to the two subsets respectively. However, as pointed out by $\mathrm{Li}$ et al. [8], MAFs have little relevance with biological information and thus partitioning SNPs based on MAFs probably may not improve much power. To address this issue, Li et al. [8] proposed a 'prioritized subset analysis' (PSA). The PSA makes clever use of available prior knowledge, either of the linkage information, the biological information or both. We will show that the PSA can greatly facilitate discoveries in GWASs, with a demonstration on an AMD data.

\section{Methods}

Materials: a GWAS on Age-related Macular Degeneration (AMD)

AMD is a genetically complex disorder. The heritability was estimated to range from $46 \%$ to $71 \%$. Klein et al. [9] reported an AMD data set containing 96 AMD cases and 50 controls. Of all the 116,204 genotyped SNPs, 99,317 SNPs were informative (MAF $\geq 1 \%$ ) and conformed to Hardy-Weinberg equilibrium (with HardyWeinberg exact $p$ value $\geq 0.05$ in the 50 controls). Following Klein et al. [9], we test for allelic association with disease status on each SNP.

\section{Prioritized Subset Analysis}

To facilitate discoveries in GWASs, we turned to a new method of 'prioritized subset analysis' (PSA) [8]. To perform a PSA, a researcher based on his/her prior biological knowledge first picks from among all SNPs under study, a certain number of SNPs likely to be the true positives. He/she then places those selected SNPs in a 'prioritized subset' and those remaining in a 'non-prioritized' subset. The FDR control is then applied to these two subsets separately, and the significant results are harvested from both the two subsets.

\section{Prioritizing SNPs}

We took findings from previous genome-wide linkage and association studies on AMD as our prior knowledge to prioritize SNPs. Our prioritization process is detailed below.

We first incorporated evidence of linkage (with LOD score $>2.0$ ) based on previous linkage studies [10-16]. We obtained the physical position of each D-number marker (listed in Table 1) from the Gene Location website http://genecards.weizmann.ac.il/geneloc/index.shtml. Then SNPs within $500 \mathrm{~kb}$ from each D-number marker were prioritized.

Moreover, several genes have had at least one positive association finding $[17,18]$. These genes with SNPs genotyped in the AMD data set are listed in Table 1. For example, complement factor $\mathrm{H}$ (CFH, GeneID 3075) gene within 1q32 was reported to be related to AMD, both from genome-wide linkage analyses [10-15] (all published before Klein et al. [9]) and case-control studies [19-21] (all independent of Klein et al.'s study [9], and published at the same year of Klein et al.'s paper [9]). We also learnt that LOC387715/HTRA1 (GeneID 5654) locus within 10q26 was identified as a second major locus contributing to AMD pathogenesis [22,23]. Furthermore, complement component factor B (CFB, GeneID 629) and the adjacent complement component 2 (C2, GeneID 717) on chromosome 6p21, were reported to act along the same biological pathway as $C F H[24,25]$. When prioritizing SNPs, we used the Bioinformatics tool 'GenoWatch' [26] to identify SNPs around these candidate genes. The chromosomal region around the $C F H$ gene had been shown by several independent studies to be linked [10-15] or associated [19-21] with AMD, so we prioritized SNPs within $1 \mathrm{Mb}$ from the $C F H$ gene. For other candidate genes in Table 1, we prioritized SNPs within $50 \mathrm{~kb}$ from each. (For example, to check whether SNP rs800292 is within 1 $\mathrm{Mb}$ from the $C F H$ gene, we simply insert 'rs800292' into 'SNP ID' and ' $1 \mathrm{Mb}$ ' into the 'Upstream' and 'Downstream' on the website of GenoWatch [26]: http://genepipe.ngc.sinica.edu.tw/genominer/menu.do)

In the end, a total of 639 SNPs were prioritized, and the remaining 98,678 SNPs, non-prioritized. We then applied the PSA with the FDR being controlled at 0.05, for both the prioritized subset and the non-prioritized

\section{Table 1 Genes or markers to be prioritized, in the prioritized subset analysis}

\begin{tabular}{lllr}
\hline Chr. & Genes & $\begin{array}{l}\text { D-number } \\
\text { markers }\end{array}$ & $\begin{array}{r}\text { No. of SNPs in the } \\
\text { prioritized subset }\end{array}$ \\
\hline 1 & ABCA4, CFH & D1S549 & 94 \\
2 & & D2S1356, & 103 \\
& & D2S1394, D2S1384 & \\
3 & CX3CR1 & D351768, & 93 \\
& & D3S1304, D3S3045 & 19 \\
4 & & D4S2368 & 169 \\
5 & & D5S820, & \\
& & GATA12A08, & \\
& & D5S1506 & \\
6 & HLA, C2-CFB, VEGF, & & 10 \\
& ELOVL4, SOD2 & & 49 \\
7 & PON1 & & 12 \\
9 & VLDLR, TLR4 & D9S930, D9S934 & 9 \\
10 & LOC387715/HTRA1 & & 32 \\
12 & LRP6 & & 17 \\
19 & APOE & D19S245 & 12 \\
20 & CST3, MMP9 & & \\
22 & & D22S683 & \\
\hline
\end{tabular}


subset. We used Storey and Tibshirani's [3] smoothing spline approach provided by the package 'fdrtool' [27] to estimate the proportions of true negative SNPs.

\section{Results}

Bonferroni Correction and Traditional FDR Approach Controlling the FWER at 0.05 (the level of significance for each SNP being set at $0.05 / 99,317=5.03 \times 10^{-7}$ with the Bonferroni correction), only one significant SNP (rs380390) can be identified (within the CFH gene, see Table 2). (Klein et al. [9] actually found one additional significant SNP, rs10272438, but it was later dropped because of low call rate and possible genotyping errors.) Controlling the FDR at 0.05 wasn't any better-the same (and the only one) SNP rs380390 was found to be significant (Table 2). Note that this SNP, rs380390, though being significant, was still not $100 \%$ guaranteed to be a true positive (because it was detected under a FDR control value of 0.05 ).

\section{Prioritized Subset Analysis}

The PSA identified a total of seven significant SNPs (all from the prioritized subset) (Table 2). These include SNPs within three genes: CFH, CFHR4, and SGCD. By using the PSA method, we have been able to detect six additional significant SNPs (in two additional genes), compared to the Bonferroni approach (the method used by Klein et al. [9]) or the traditional FDR approach. Two of the three significant genes found in this study, CFH and CFHR4, are located in a chromosomal region (1q31-1q32) having been most replicated in previous AMD studies. The remaining one significant $S G C D$ gene had not been previously reported to be AMDrelated, though. However, we notice that previous animal studies showed the $S G C D$ gene is related to vascular abnormalities in mice [28]. This might suggest a link of $S G C D$ to neovascular AMD in humans.

All the seven significant SNPs are from the prioritized subset. To evaluate how well the FDR is controlled in our prioritized subset, we further estimated the permutation-based FDR [29] in this subset. We randomly permuted the data and calculated the null $P$ values $p_{i}^{(b)}$ for the $i$ th SNP in the $b$ th permutation $(i=$ $1, \ldots, 639)$. Through $B$ permutations, the number of false positives $(F P)$ is estimated as $\hat{F P}(d)=\sum_{b=1}^{B} \#\left\{i: p_{i}^{(b)} \leq d\right\} / B$, where $d=5.12 \times 10^{-4}$ is the largest $P$ value of the seven significant SNPs (see Table 2). We took $B=100,000$ and obtained $\hat{F P}=0.225$. The permutation-based FDR in the prioritized subset is thus estimated as $0.225 / 7=$ 0.032 , which is still less than our FDR control level of 0.05 , suggesting a satisfactory FDR control in this subset.

\section{Discussion}

Prior information can come from a researcher's biological knowledge, or findings of data other than that provided in the current study. But one should not 'snoop' his/her data at hand for the prior knowledge. If one naively prioritizes those SNPs with the smallest $p$ values in the study data, the actual overall FDR would no longer be properly controlled. To avoid such bias, we searched findings of other data to build our 'prior knowledge', before seeing the analysis results of individual SNPs in the current AMD data set. At that time, we did know that rs380390 is a significant SNP in the AMD data set which can withstand a FWER control of 0.05 [9]. But the chromosomal region around rs 380390 had already been replicated by many previous linkage studies [10-15] (all published before Klein et al. [9]). And so, prioritizing chromosomal region around rs380390 won't constitute an act of data snooping.

Around a particular gene, how large a chromosomal region should be prioritized is also an issue. Because of the consistent findings in the $\mathrm{CFH}$ gene, both from genome-wide linkage analyses [10-15] and case-control studies [19-21], we prioritized SNPs within $1 \mathrm{Mb}$ from the $\mathrm{CFH}$ gene. Other evidence of linkage and associations are relatively unconfirmed by prior studies, so we prioritized SNPs within $500 \mathrm{~kb}$ and $50 \mathrm{~kb}$, respectively. Because linkage is a coarse mapping whereas association is a fine mapping, in general a wider region of SNPs should be prioritized for a linkage peak. Admittedly,

Table 2 Results of the AMD data set

\begin{tabular}{|c|c|c|c|c|c|c|c|}
\hline Chr. & Location (bp) & SNP & $P$ value $*$ & Bonferroni & FDR & PSA & Gene \\
\hline 1 & 193930492 & rs800292 & $5.12 \times 10^{-4}$ & NS ** & NS & $S$ & $\mathrm{CFH}$ \\
\hline 1 & 193962973 & rs2019727 & $3.01 \times 10^{-4}$ & NS & NS & $\mathrm{S}$ & \\
\hline 1 & 193989310 & rs380390 & $5.40 \times 10^{-8}$ & S & S & S & \\
\hline 1 & 193991069 & rs1329428 & $3.09 \times 10^{-6}$ & NS & NS & $S$ & \\
\hline 1 & 194173603 & rs1853882 & $1.59 \times 10^{-4}$ & NS & NS & $\mathrm{S}$ & CFHR4 \\
\hline 5 & 155782975 & rs970476 & $7.20 \times 10^{-5}$ & NS & NS & $\mathrm{S}$ & SGCD \\
\hline 5 & 155791718 & rs931798 & $3.69 \times 10^{-4}$ & NS & NS & $S$ & \\
\hline
\end{tabular}

* $P$ values were obtained from Fisher's exact test for allelic association with disease status.

** S: significant; NS: not significant 
there is no absolute criterion for choosing the sizes of prioritized regions. No matter how large a chromosomal region is prioritized, the FDR within subsets should be controlled at the desired level, and this can be verified by estimating the permutation-based FDR [29].

In recent GWASs, a commonly used approach to incorporate prior knowledge is to calculate the Bayes factors $[1,30]$. However, to estimate the Bayes factors, the prior distributions and the effect sizes should be carefully specified [30]. This may limit its applicability. By contrast, the PSA method used in this paper can feed on prior knowledge that is only rudimentary (we need only to decide beforehand whether a particular SNP is more likely a true positive or a true negative, but don't need to know exactly how likely). And there is almost no penalty for poor guessing [8]. In this paper, we demonstrated that such a simple dichotomization followed by a simple PSA can greatly facilitate discoveries in a GWAS on AMD.

Note that we did not recruit any more subjects or type any more SNPs beyond what Klein et al. [9] had done. The only thing we did is to incorporate prior knowledge about AMD into the analysis. And we see this input of knowledge is rather powerful (six/two additional significant SNPs/genes were identified in the same AMD casecontrol data). One may question that our input of knowledge and the subsequent partition of SNPs into two subsets to be tested separately and harvested combinedly are making easier (and perhaps too easier) for the SNPs to come out. But we should emphasize that we did not loosen our FDR control in any way. The total seven significant SNPs found in this re-analysis have an overall 0.05 FDR attached to them, much the same way with the one SNP rs380390 originally found in Klein et al. [9] had a 0.05 FDR attached to it. And we believe that researchers will find no difficulties to choose seven SNPs or just one-that is, under the same FDR criteria.

\section{Conclusions}

The PSA approach is rather powerful and is easy to implement. Based on the success of our re-analysis of Klein et al's GWAS on AMD, we advocate using PSA to facilitate discoveries in future GWASs.

\begin{abstract}
Acknowledgements
We would like to thank the anonymous reviewers for their constructive comments. We also thank Dr. Josephine Hoh for kindly providing the AMD data set. This study was supported by National Science Councils, Taiwan.
\end{abstract}

\section{Author details}

${ }^{1}$ Graduate Institute of Epidemiology, College of Public Health, National Taiwan University, Taipei, Taiwan. ${ }^{2}$ Research Center for Genes, Environment and Human Health, National Taiwan University, Taipei, Taiwan.

\section{Authors' contributions}

$W-Y L$ participated in the design of the study, performed the data analyses, and drafted the manuscript. W-C L conceptualized the study, provided advice, and revised the manuscript. All authors read and approved the final manuscript.

\section{Competing interests}

The authors declare that they have no competing interests.

Received: 15 December 2009

Accepted: 28 January 2010 Published: 28 January 2010

\section{References}

1. WTCCC: Genome-wide association study of 14,000 cases of seven common diseases and 3,000 shared controls. Nature 2007, 447(7145):661-678.

2. Benjamini Y, Hochberg Y: Controlling the false discovery rate: A practical and powerful approach to multiple testing. J R Stat Soc B 1995, 57:289-300.

3. Storey JD, Tibshirani R: Statistical significance for genomewide studies. Proc Natl Acad Sci USA 2003, 100(16):9440-9445.

4. Holm S: A simple sequentially rejective multiple test procedure. Scand J Statist 1979, 6:65-70.

5. Genovese C, Roeder K, Wasserman L: False discovery control with P-value weighting. Biometrika 2006, 93:509-524.

6. Roeder K, Bacanu SA, Wasserman L, Devlin B: Using linkage genome scans to improve power of association in genome scans. Am J Hum Genet 2006, 78(2):243-252.

7. Sun L, Craiu RV, Paterson AD, Bull SB: Stratified false discovery control for large-scale hypothesis testing with application to genome-wide association studies. Genet Epidemiol 2006, 30(6):519-530.

8. Li C, Li M, Lange EM, Watanabe RM: Prioritized subset analysis: improving power in genome-wide association studies. Hum Hered 2008, 65(3):129-141.

9. Klein RJ, Zeiss C, Chew EY, Tsai JY, Sackler RS, Haynes C, Henning AK, SanGiovanni JP, Mane SM, Mayne ST, et al: Complement factor $\mathrm{H}$ polymorphism in age-related macular degeneration. Science 2005, 308(5720):385-389.

10. Abecasis GR, Yashar BM, Zhao Y, Ghiasvand NM, Zareparsi S, Branham KE, Reddick AC, Trager EH, Yoshida S, Bahling J, et al: Age-related macular degeneration: a high-resolution genome scan for susceptibility loci in a population enriched for late-stage disease. Am J Hum Genet 2004, 74(3):482-494.

11. Iyengar SK, Song D, Klein BE, Klein R, Schick JH, Humphrey J, Millard C, Liptak R, Russo K, Jun G, et al: Dissection of genomewide-scan data in extended families reveals a major locus and oligogenic susceptibility for age-related macular degeneration. Am J Hum Genet 2004, 74(1):20-39.

12. Klein ML, Schultz DW, Edwards A, Matise TC, Rust K, Berselli CB, Trzupek K, Weleber RG, Ott J, Wirtz MK, et al: Age-related macular degeneration. Clinical features in a large family and linkage to chromosome 1q. Arch Ophthalmol 1998, 116(8):1082-1088.

13. Majewski J, Schultz DW, Weleber RG, Schain MB, Edwards AO, Matise TC, Acott TS, Ott J, Klein ML: Age-related macular degeneration-a genome scan in extended families. Am J Hum Genet 2003, 73(3):540-550.

14. Seddon JM, Santangelo SL, Book K, Chong S, Cote J: A genomewide scan for age-related macular degeneration provides evidence for linkage to several chromosomal regions. Am J Hum Genet 2003, 73(4):780-790.

15. Weeks DE, Conley YP, Tsai HJ, Mah TS, Schmidt S, Postel EA, Agarwal A, Haines JL, Pericak-Vance MA, Rosenfeld PJ, et al: Age-related maculopathy: a genomewide scan with continued evidence of susceptibility loci within the 1q31, 10q26, and 17q25 regions. Am J Hum Genet 2004, 75(2):174-189

16. Jun G, Klein BE, Klein R, Fox K, Millard C, Capriotti J, Russo K, Lee KE, Elston RC, lyengar SK: Genome-wide analyses demonstrate novel loci that predispose to drusen formation. Invest Ophthalmol Vis Sci 2005, 46(9):3081-3088

17. Scholl HP, Fleckenstein M, Charbel Issa P, Keilhauer C, Holz FG, Weber BH: An update on the genetics of age-related macular degeneration. Mol Vis 2007, 13:196-205. 
18. Haddad S, Chen CA, Santangelo SL, Seddon JM: The genetics of agerelated macular degeneration: a review of progress to date. Surv Ophthalmol 2006, 51(4):316-363.

19. Edwards AO, Ritter R, Abel K, Manning A, Panhuysen C, Farrer LA: Complement factor $\mathrm{H}$ polymorphism and age-related macular degeneration. Science 2005, 308(5720):421-424.

20. Haines JL, Hauser MA, Schmidt S, Scott WK, Olson LM, Gallins P, Spencer KL, Kwan SY, Noureddine M, Gilbert JR, et al: Complement factor $\mathrm{H}$ variant increases the risk of age-related macular degeneration. Science 2005, 308(5720):419-421.

21. Hageman GS, Anderson DH, Johnson LV, Hancox LS, Taiber AJ, Hardisty LI, Hageman JL, Stockman HA, Borchardt JD, Gehrs KM, et al: A common haplotype in the complement regulatory gene factor $\mathrm{H}$ ( $\mathrm{HF} 1 / \mathrm{CFH})$ predisposes individuals to age-related macular degeneration. Proc Natl Acad Sci USA 2005, 102(20):7227-7232.

22. Rivera A, Fisher SA, Fritsche $L G$, Keilhauer $C N$, Lichtner $P$, Meitinger $T$, Weber BH: Hypothetical LOC387715 is a second major susceptibility gene for age-related macular degeneration, contributing independently of complement factor $\mathrm{H}$ to disease risk. Hum Mol Genet 2005, 14(21):3227-3236.

23. Jakobsdottir J, Conley YP, Weeks DE, Mah TS, Ferrell RE, Gorin MB: Susceptibility genes for age-related maculopathy on chromosome 10q26. Am J Hum Genet 2005, 77(3):389-407.

24. Gold B, Merriam JE, Zernant J, Hancox LS, Taiber AJ, Gehrs K, Cramer K, Neel J, Bergeron J, Barile GR, et al: Variation in factor B (BF) and complement component 2 (C2) genes is associated with age-related macular degeneration. Nat Genet 2006, 38(4):458-462.

25. Jakobsdottir J, Conley YP, Weeks DE, Ferrell RE, Gorin MB: C2 and CFB genes in age-related maculopathy and joint action with $\mathrm{CFH}$ and LOC387715 genes. PLOS ONE 2008, 3(5):e2199.

26. Chen YH, Liu CK, Chang SC, Lin YJ, Tsai MF, Chen YT, Yao A: GenoWatch: a disease gene mining browser for association study. Nucleic Acids Res 2008, , 36 Web Server: W336-340.

27. Strimmer $\mathrm{K}: \mathrm{A}$ unified approach to false discovery rate estimation. $B M C$ Bioinformatics 2008, 9:303.

28. Dye WW, Gleason RL, Wilson E, Humphrey JD: Altered biomechanical properties of carotid arteries in two mouse models of muscular dystrophy. J Appl Physio/ 2007, 103(2):664-672.

29. Xie Y, Pan W, Khodursky AB: A note on using permutation-based false discovery rate estimates to compare different analysis methods for microarray data. Bioinformatics 2005, 21(23):4280-4288.

30. Wakefield J: Reporting and interpretation in genome-wide association studies. Int J Epidemiol 2008, 37(3):641-653.

\section{Submit your next manuscript to BioMed Central and take full advantage of:}

- Convenient online submission

- Thorough peer review

- No space constraints or color figure charges

- Immediate publication on acceptance

- Inclusion in PubMed, CAS, Scopus and Google Scholar

- Research which is freely available for redistribution 